\title{
Coordination Methods of Thioligands in Trinuclear Complexes of Platinum (II)
}

\author{
KHUDAYAR GASANOV, SELJAN NURULLAYEVA, ZIYA BABAYEV \\ Azerbaijan Medical University \\ AZERBAIJAN, BAKU
}

\begin{abstract}
The trinuclear complexes with simple and mixed ligands of platinum (II) with mercaptoethanol and mercaptoethylamine have been generated and their contents and structures investigated by numerous physicochemical methods. Mercaptoethanol ( $\mathrm{SHCH} 2 \mathrm{CH} 2 \mathrm{OH})$ is coordinated monodentally with just sulfur atom and forms pentacyclic metalchelate ring, where as mercaptoethylamine ( $\mathrm{HSCH} 2 \mathrm{CH} 2 \mathrm{NH} 2)$ is coordinated monodentally as well as bidentally and forms an identical ring (pentacyclic metalchelate). The sulfur atom of ligands plays a bridge role between the metals in the trinuclear platinum complexes. The hydroxyl group of mercaptoethanol does not participate in the coordination.
\end{abstract}

Key-Words: mercaptoethanol, mercaptoethylamine, platinum, trinuclear, metalchelate cycle, bridge, terminal.

\section{INTRODUCTION}

The great interest in ligands containing sulfur hydroxyl, sulfur amine groups is related to their containing functional groups that belong to biological systems. In the other hand, it is possible to generate coordination compounds that are variously resistant with coordination sphere purposively synthesizing ligands with platinum salts [1].

As an investigating object, mercaptoethanol $\mathrm{HSCH} 2 \mathrm{CH} 2 \mathrm{OH}$ and mercaptoethylamine $\mathrm{HSCH} 2 \mathrm{CH} 2 \mathrm{NH} 2$ ligands containing sulfur-oxygen, sulfur-nitrogen donor atoms have been taken. These ligands have universal effect and form very stable and soluble in water complex compounds that are able to solve some problems of the science, technology and medicine [2]. There are reference informations about biological activity of the complex compounds of the ligands and their some derivations containing sulfur, oxygen and nitrogen donor atoms [3]. In the proffered work trinuclear complexes with simple and mixed ligands have been synthesized with palladium and investigated. Complexes with simple and mixed ligands have been synthesized and studied comparatively considering that the polynuclear mixed complexes of palladium are technically and medically important.

During experimentation 2-mercaptoethanol $\mathrm{HSCH} 2 \mathrm{CH} 2 \mathrm{OH}$ and 2-mercaptoethylamine (mercamine) - $\mathrm{HSCH} 2 \mathrm{CH} 2 \mathrm{NH} 2$ produced by "Ferak" and "Serva" companies, respectively, have been used without decontamination. The IR-spectra of the synthesized complexes and ligands were drawn in "Thermoscientific, Nicoltis 10" and "Bruker IFS-113V" spectrometers, in vaseline suspension, fluorinated oil and pill-shaped $\mathrm{KBr}$ (in spectrum diapason of $200-500,400-4000 \mathrm{~cm}-1$ ). The electroconductivities of the complexes in aqueous or aqueous-alcohol solutions were measured by "KEL$1 \mathrm{M} 2$ " conductometer at $250 \mathrm{C}$. Thermical resistance and reactions were investigated in "STA 449 F3 Yupiter" derivatograph of "NETZSCH" company heating $100 \mathrm{C}$ in every minute. The element analysis of the synthesized complexes was carried out with "CHNSOE Carlo ERBA" analyzer.

\section{Synthesis of \\ [Pt3(SCH2CH2OH)4(NH3)2Cl2].2H2O} complex (I).

$0,60 \mathrm{~g}(1,09 \mathrm{mmol})$ of $\mathrm{K} 2[\mathrm{PtCl} 4]$ salt was dissolved in $20 \mathrm{~mL}$ of $\mathrm{NH} 4 \mathrm{OH}$ solution in compact flask regularly stirring at $550 \mathrm{C}$ and filtered through a paper filter to remove partially reduced metallic platinum. $0,50 \mathrm{~g} \quad(6,35 \mathrm{mmol})$ of ligands mercaptoethanol $10 \%$ more than a stoichiometric ratio was added to filtered hot (450C) solution. Reaction mixture $(\mathrm{pH}=8,5)$ was continuously heated in compact flask for 3 hours at 55-650C. The dark pink complex compound was formed from obtaining same-colored solution. The complete precipitation process proceeds for 2 days at 60C. The precipitation was filtered and washed with cold water, alcohol and ester. The obtained substance first was dried at room temperature, then in a 
vacuum over $\mathrm{CaCl} 2$ till it got a stable weight. Yield was $0,69 \mathrm{~g}(46 \%)$.

For C8H30S4N2Cl2O6Pt3 formula:

$\mathrm{Pt}-56,31 ; \mathrm{S}-12,18 ; \mathrm{N}-2,56 ; \mathrm{Cl}-6,63 ; \mathrm{C}-$ 9,$11 ; \mathrm{H}-2,73$ were found.

$\mathrm{Pt}-56,57 ; \mathrm{S}-12,39 ; \mathrm{N}-2,70 ; \mathrm{Cl}-6,85 ; \mathrm{C}-$ 9,28; $\mathrm{H}-2,90$ were calculated.

The compound dissolves well enough in hot water and alcohol.

\section{Synthesis of}

[Pt3(SCH2CH2NH2)2(SCH2CH2OH)2(NH3)2 Cl2] complex (II).

$0,36 \mathrm{~g}$ $(0,37 \mathrm{mmol})$

of

[Pt3(SCH2CH2NH2)4]Cl2.H2O complex compound obtained by knowing the way [4] was taken and prepared suspension mixing in $20 \mathrm{~mL}$ of hot $\mathrm{NaOH}$ solution. This suspension dissolved when reaction mixture was stirred at $550 \mathrm{C}$ for 40 minutes and it changed into transparent solution. $0,09 \mathrm{~g}$ $(1,15 \mathrm{mmol})$ of mercaptoethanol $\mathrm{HSCH} 2 \mathrm{CH} 2 \mathrm{OH}$ dissolved in $10 \mathrm{~mL}$ water was added into reaction mixture, as a result of that light pink precipitation was formed. Reaction proceeded $(\mathrm{pH}=9)$ for 1,5 hours at $450 \mathrm{C}$. When precipitation process finished completely the compound was filtered and removed from solution and washed first with cold water, then with alcohol and ester. Obtained complex compound was dried in vacuum over $\mathrm{CaCl} 2$ until it got a stable weight. Yield was $0,28 \mathrm{~g}(76 \%)$.

For C8H28N4S4Cl2O2Pt3 formula:

$\mathrm{Pt}-58,56 ; \mathrm{S}-12,68 ; \mathrm{N}-5,49 ; \mathrm{Cl}-6,92 ; \mathrm{C}-$ 9,57; $\mathrm{H}-2,73$ were found.

Pt- 58,73; S - 12,86; N - 5,61; Cl - 7,11; C 9,64; $\mathrm{H}-2,80$ were calculated.

The compound dissolves well enough in hot water and alcohol.

\section{Synthesis of}

[Pt3(SCH2CH2NH2)2(SCH2CH2OH)2Cl2] complex (III).

$0,32 \mathrm{~g}$ of complex II was transferred into compact flask, $20 \mathrm{~mL}$ of water and $10 \mathrm{~mL}$ of $15 \% \mathrm{KOH}$ solution added and regularly stirred for 1,5 hours at 650C. Ultimately, the light pink colour of reaction mixture was changed into dark yellow $(\mathrm{pH}=9,5)$, then transparent solution filtered and evaporated at $500 \mathrm{C}$ in water-bath until little volume. Obtained syrupy mass was treated with $70 \mathrm{~mL}$ chloroform at first, then the exess amount of solvent dismissed, the yellowish compound precipitated in this step. Precipitation was filtered and washed with cold alcohol and ester. Formed compound was dried over $\mathrm{CaCl} 2$ until a stable weight. Yield is $0,24 \mathrm{~g}(83 \%)$.

For $\mathrm{C} 8 \mathrm{H} 22 \mathrm{~N} 2 \mathrm{~S} 2 \mathrm{Cl} 2 \mathrm{O} 2 \mathrm{Pt} 3$ formula:

$\mathrm{Pt}-65,39 ; \mathrm{S}-7,26 ; \mathrm{N}-3,35 ; \mathrm{Cl}-7,58 ; \mathrm{C}-$ 10,$47 ; \mathrm{H}-2,22$ were found.

$\mathrm{Pt}-65,15 ; \mathrm{S}-7,14 ; \mathrm{N}-3,12 ; \mathrm{Cl}-7,88 ; \mathrm{C}-$ 10,$69 ; \mathrm{H}-2,45$ were calculated.

This substance dissolves well in water and alcohol.

\section{ANALYSIS OF THE OUTCOMES}

Mercaptoethanol and mercaptoethylamine are soft hydroxides and contain not only $\mathrm{OH}$ and $\mathrm{NH} 2$ groups, but also polar HS group has the strong reaction ability. A sulfohydryl group differs bitterly from trial $>\mathrm{S}$ functional group with respect its reaction ability. Therefore, it can be presumed that complexes with simple and mixed ligands formed with palladium and ligands containing sulfohydryl group have different contents, structures, resistances and other properties.

The ratios, medium, solvents, temperature and other factors of synthesis are optimal. The high yield of pure complex compounds

[Pt3(SCH2CH2OH)4(NH3)2Cl2].2H2O

(I),

[Pt3(SCH2CH2NH2)2(SCH2CH2OH)2(NH3)2Cl2]

\section{[Pt3(SCH2CH2NH2)2(SCH2CH2OH)2Cl2]}

is acquired only under above-mentioned condition seriously supervising.

To get whole results about the structures of complexes IR-spectra of the ligands and newly synthesized coordination compounds have been studied and assayed comparatively. SH functional groups belong to both ligands - mercaptoethanol and mercaptoethylamine are characterized by 2568 and $2562 \mathrm{~cm}-1$ adsorption bands in IR-spectra, respectively. These adsorption bands are identical to reference materials [4,5] and any adsorption band pertaining to HS-group was not recorded in IRspectra of all synthesized I-III complexes. And it implicitly indicates that HS group is deprotonated with central atom and forms vPt-S valence bond. 288, 294 and $330 \mathrm{~cm}-1$ adsorption bands were recorded within 200-400 cm-1 adsorption zone in IR-spectrum of complex I. First two adsorption 
bands $v_{P t-S}^{\text {koord. }}$ belong to bridge-shaped Pt-S valence bond. Other strong $330 \mathrm{~cm}-1$ adsorption band belongs to $v_{P t-C l}^{\text {ter. }}$ terminal bond [6]. The first two adsorption bands from 284, 286, 290 and $292 \mathrm{~cm}-1$ partially slid and clearly expressed in IR-spectrum of complex II and are close to above-mentioned adsorption bands belong to mercaptoethanol that forms valence bond in Pt-S bridge position, the other two bands belong to mercaptoethylamine $\mathrm{Pt}-\mathrm{S}$ valence bond [7]. Other ledgeless 328 cm-1 adsorption band belongs to $v_{P t-C l}^{t e r}$ terminal valence bond.

When complex II is treated in alkaline medium ammonia is ejected from coordination sphere by amine group of mercaptoethylamine and as a result of that trinuclear metalchelate complex III is formed. In consequence of chelateformation the innercomplex electronic density distributed samely among atoms and the adsorption bands in IR-spectrum of complex appear clearly. Three slightly slipped adsorption bands - 262, 276 and $293 \mathrm{~cm}-1$ - of Pt-S bond indicate innercomplex high spin splitting and low spin splitting, in other words destroying its flat plane structure. Three adsorption bands of platinum sulfur bridge

$$
>\mathrm{Pt}^{-}>\mathrm{S} \backslash \mathrm{S}>\mathrm{Pd}<
$$

bond show that sulfur atom has pyramidal structure, and this makes its configuration "armchair-shaped" [8]. Expression of Me-S valence bond in bridge position with three adsorption bands indicates that complex has spin configuration. This case is known from reference source [9].

The complexed and complicated adsorption bands appear within 2400-3700 cm-1 adsorption zone in IR-spectra of complexes I-II, it means that these complexes have hydrogen bonds like $\mathrm{OH}$..... $\mathrm{NH} 2 \ldots \mathrm{Cl}$. On the other hand, the adsorption bands belonging to $\mathrm{OH}$ and $\mathrm{NH} 2$ groups of the ligands were not expressed in IRS of the complexes. Such situation is often faced in references [10].

The high intensive $\delta(\mathrm{N}-\mathrm{H})$ adsorption bands observed in IR-spectra of complex I (II) probably belong to $\mathrm{N}-\mathrm{H}$ deformative bond and $\mathrm{Pt}-\mathrm{N}$ valence bond. The expressions demonstrating mentioned adsorption bands find place in reference [11].
As a result of forming of pentacyclic

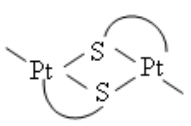

metalchelate ring in bridge form and of coordinating of mercaptoethylamine bidentally the Pt-N in terminal position is characterized by two -371 and $382 \mathrm{~cm}-1$ adsorption bands in IRS of valence bond unlike complexes I and II [12,13].

The hydrogen bonds existing between $\mathrm{NH} . . . \mathrm{Cl}$ and $\mathrm{H} 2 \mathrm{O} \ldots \mathrm{Cl}$ in IR-spectra of complexes I-III stabilize the structures of the complexes, and this prompts structural changes not to happen in aqueous solution of the complexes.

The numerical values $(40,52,47$ Om$1 \mathrm{~cm} 2 \mathrm{~mol}-1)$ of electroconductivities of all three complexes in 10-3M aqueous and aqueous-alcohol solution demonstrate that these complexes are nonelectrolates and the estimated formulas for them are correct.

Thermical decomposition of complexes I-III is different from each other depending on the ligand surroundings of central atom. Thus, the thermical decomposition of complex I takes place in two steps. In first step, in other words, at $1650 \mathrm{C}$ dehydration happens, the second step is ended with decomposition of complex without melting at 2900C. Complex II is decomposed at $2700 \mathrm{C}$, III at 3200C without melting. The last product of thermolysis of all three complexes is PtO.

$[\mathrm{Pt}(\mathrm{SCH} 2 \mathrm{CH} 2 \mathrm{OH}) 4 \mathrm{Cl} 2(\mathrm{NH} 3) 2] .2 \mathrm{H} 2 \mathrm{O})$

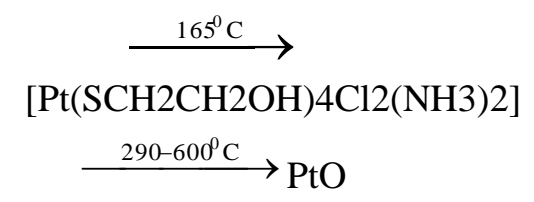

In complex III with mixed ligand mercaptoethylamine is coordinated bidentally and forms two symmetrical pentacyclic metalchelate ring, therefore it is decomposed in higher temperature than its analogues.

The antimicrobial properties of every complex have been learned comparatively. The complex I unlike other complexes indicates strong antimicrobial property. The microbial properties decrease according to I $>$ II $>$ III order. 


\section{CONCLUSION}

It has been demonstrated by modern physicochemical ways that newly synthesized complexes with simple and mixed ligands containing sulfur-oxygen, sulfur-nitrogen donor atom are trinuclear.

In complex I four mercaptoethanol molecules from sulfur atoms are coordinated monodentally with platinum in bridge position. In complex II mercaptoethanol and mercaptoethylamine are coordinated monodentally and form coordination compounds with mixed ligand.

In complex III mercaptoethanol is coordinated monodentally, mercaptoethylamine is coordinated forming pentacyclic metalchelate ring, namely complex compounds with mixed ligands and mixed coordination.

\section{References:}

[1] H. Sakurai, Y. Kojima, Y. Yoshikawa, K. Kawabe, H. Yasui.// Coord. Chem. Rev. 2002 (226) p.187-198

[2] 2.A.I. Matesanz, P. Souza.// J. Inor. Biochem. 2007 (101) p. 1354-1361.

[3] 3. T. Rosu, E. Pahontu, S. Pasculescu, R. Georgescu, N. Stanica, A. Curaj,

[4] A. Popescu, M. Leabu. //Eur. J. Med. Chem. 2010 ( 45 ) p. 1627-1634.

[5] 4. Hasanov Kh.I., Fatullayeva S.S., Tagiev D.B., Mirzai J.I.// J. Azerb. Chem.. 1998. №3. p. $38-42$.

[6] 5. Yefimenko I.A., Hasanov Kh.I., Gorbunova Y.Y. //Pres. RAN. 1992.(326)p. 654-660.

[7] 6. Fedorova O.V., Koryanova O.V., Rusinova G.L. // General Chemistry J. 2000. p.1654-1657.

[8] 7. D. Gambino, L. Otero, M. Vieites, M. Boiani, M. Gonzalez, E.J. Baran, H. Cerecetto, Spectrochim. Acta.// A 2007( 68) p.341-348.

[9] 8. D. Vazquez-Garcia, A. Fernandez, J.J. Fernandez, M. Lopez-Torres, A. Suarez, J.M. Ortigueira, J.M. Vila, H. Adams.// J. Organomet. Chem. 2000 (595 ) p. 199-207.

[10] 9. V. Suni, M.R.P. Kurup, M. Nethaji, Spectrochim.// Acta Part A 2006 (63) p.174181.

[11] 10. A.P. Rebolledo, M. Vieites, D. Gambino, O.E. Piro, E.E. Castellano, C.L. Zani, E.M. Souza-Fagundes, L.R. Teixeira, A.A. Batista,

\section{H. Beraldo.// J. Inorg. \\ Biochem. 2005(} 99 ) p.698-706.

[12] 11. L. Papathanasis, M.A. Demertzis, P.N. Yadav, D. Kovala-Demertzi, C. Prentjas, A. Castineiras, S. Skoulika, D.X. West.// Inorg. Chim. Acta 2004 ( 357) p. 4113-4120.

[13] 12. A. Castineiras, S. Skoulika, D.X. West.// Inorg. Chim. Acta 2004 (357) p. 4113-4120.

[14] 13. R.A. Alderden, M.D. Hall, T.W. Hambley.// J. Chem. Ed. 2006 (83) 728-734. 